\title{
Face Recognition by Discriminant Analysis with Gabor Tensor Representation
}

\author{
Zhen Lei, Rufeng Chu, Ran He, Shengcai Liao, and Stan Z. Li \\ Center for Biometrics and Security Research \& National Laboratory of Pattern Recognition, \\ Institute of Automation, Chinese Academy of Sciences, \\ 95 Zhongguancun Donglu, Beijing 100080, China \\ \{zlei, rfchu, rhe, scliao, szli\}@nlpr.ia.ac.cn \\ http://www.cbsr.ia.ac.cn
}

\begin{abstract}
This paper proposes a novel face recognition method based on discriminant analysis with Gabor tensor representation. Although the Gabor face representation has achieved great success in face recognition, its huge number of features often brings about the problem of curse of dimensionality. In this paper, we propose a 3rd-order Gabor tensor representation derived from a complete response set of Gabor filters across pixel locations and filter types. 2D discriminant analysis is then applied to unfolded tensors to extract three discriminative subspaces. The dimension reduction is done in such a way that most useful information is retained. The subspaces are finally integrated for classification. Experimental results on FERET database show promising results of the proposed method.
\end{abstract}

Keywords: discriminant analysis, Gabor tensor representation, face recognition.

\section{Introduction}

Face images lie in a highly nonlinear and nonconvex manifolds in the image space due to the various changes by expression, illumination and pose etc. Design a robust and accurate classifier in such a nonlinear and nonconvex distribution is difficult work. One approach to simplify the complexity is to construct a local appearance-based feature space, using appropriate image filters, so that the distributions of faces are less affected by various changes. Gabor wavelet-based features have been used for this purpose [5[14|8].

The Gabor wavelets, whose kernels are similar to the two-dimensional (2D) receptive field profiles of the mammalian cortical simple cells, exhibit desirable characteristics of spatial locality and orientation selectivity. It is robust to variations due to expression and illumination changes and is one of the most successful approaches for face recognition. However, Gabor features are usually very high-dimensional data and there are redundancies among them. It is well known that face images lie in a manifold of intrinsically low dimension; therefore, the Gabor feature representations of faces could be analyzed further to extract the underlying manifold by some statistical approach such as subspace methods. 
Subspace methods such as PCA, LDA [113] have been extensively studied in face recognition research. PCA uses the Karhunen-Loeve transform to produce the most expressive subspace for face representation and recognition by minimizing the residua of the reconstruction. However, it does not utilize any class information and so it may drop some important clues for classification. LDA is then proposed and it seeks subspace of features best separating different face classes by maximizing the ratio of the betweenclasses scatter to the within-class scatter. It is an example of the most discriminating subspace methods.

However, because of the usually high dimensions of feature space (e.g. the total number of pixels in an image) and small sample size, the within-class scatter matrix $S_{w}$ is often singular, so the optimal solution of LDA cannot be found directly. Therefore, some variants of LDA have been proposed such as PCA+LDA (Fisher-LDA), Direct LDA (D-LDA), Null space LDA (N-LDA) [1[192] etc. However, these LDAs all try to solve the singular problem of $S_{w}$ instead of avoiding it. None of them can avoid losing some discriminative information helpful for recognition. Recently, Two-Dimensional Linear Discriminant Analysis (2D-LDA) method [186] has been discussed as a generalization of traditional 1D-LDA 1 The main idea of the $2 \mathrm{D}$ method is to construct the scatter matrices using image matrices directly instead of vectors. As the image scatter matrices have a much smaller size, 2D-LDA significantly reduces the computational cost and avoids the singularity problem. Further generalization has also been proposed to represent each object as a general tensor of second or higher order, such as PCA and discriminant analysis with tensor representation [15[16].

Previous work usually [3]9]12] performs subspace analysis with Gabor vector representation. Because of the high-dimension of Gabor feature vectors and the scarcity of available data, it leads to the curse of dimensionality problem. Moreover, for objects such as face images, a vector representation ignores higher-order structures in the data. Yan et al. [16] proposed discriminant analysis with tensor representation. It encoded an object as a general tensor and iteratively learned multiple interrelated subspaces for obtaining a lower-dimensional space. However, the computational convergence of the method is not guaranteed and it is hard to extend it to kernel form.

In this paper, we propose a novel discriminant analysis method with Gabor tensor representation for face recognition. Compared to the method in [16], we introduce an alternative way for feature extraction on Gabor tensor in such a way that we can derive a non-iterative way for discrimination and extend it to kernel learning easily. The algorithm is divided into three steps. First, a face image is encoded by Gabor filters to form a 3rd-order tensor; Second, the 3rd-order tensor is unfolded into three 2nd-order tensors and 2D linear/kernel discriminant analysis is conducted on them respectively to derive three discriminative subspaces; and finally these subspaces are integrated and reduced further for classification with a nearest neighbor classifier.

The rest of the paper is organized as follows: Section 2 describes the Gabor tensor representation. Section 3 details the discriminant analysis method with Gabor tensor representation. The experimental results on FERET database are demonstrated in Section 4 and in Section 5, we conclude the paper.

\footnotetext{
${ }^{1}$ LDA based on vectors is noted as 1D-LDA.
} 


\section{Gabor Tensor Representation}

The representation of faces using Gabor features has been extensively and successfully used in face recognition [14]. Gabor features exhibit desirable characteristics of spatial locality and orientation selectively and optimally localized in the space and frequency domains. The Gabor kernels are defined as follows:

$$
\psi_{\mu, v}=\frac{k_{\mu, v}^{2}}{\sigma^{2}} \exp \left(-\frac{k_{\mu, v}^{2} z^{2}}{2 \sigma^{2}}\right)\left[\exp \left(i k_{\mu, \nu} z\right)-\exp \left(-\frac{\sigma^{2}}{2}\right)\right]
$$

where $\mu$ and $v$ define the orientation and scale of the Gabor kernels respectively, $z=$ $(x, y)$, and the wave vector $k_{\mu, v}$ is defined as follows:

$$
k_{\mu, v}=k_{\nu} e^{i \phi_{\mu}}
$$

where $k_{v}=k_{\max } / f^{v}, k_{\max }=\pi / 2, f=\sqrt{2}, \phi_{\mu}=\pi \mu / 8$. The Gabor kernels in (1) are all self-similar since they can be generated from one filter, the mother wavelet, by scaling and rotating via the wave vector $k_{\mu, v}$. Each kernel is product of a Gaussian envelope and a complex plane wave, while the first term in the square brackets in (1) determines the oscillatory part of the kernel and the second term compensates for the DC value. Hence, a band of Gabor filters is generated by a set of various scales and rotations of the kernel.

In this paper, we use Gabor kernels at five scales $v \in\{0,1,2,3,4\}$ and eight orientations $\mu \in\{0,1,2,3,4,5,6,7\}$ with the parameter $\sigma=2 \pi[9]$ to derive the Gabor representation by convoluting face images with corresponding Gabor kernels. For every image pixel we have totally 40 Gabor magnitude coefficients which can be regarded as a Gabor feature vector of 40 dimensions. Therefore, a $h \times w 2 \mathrm{D}$ image can be encoded by 40 Gabor filters to form a $40 \times h \times w 3$ rd-order Gabor tensor. Fig. 11 shows an example of a face image with its corresponding 3rd-order Gabor tensor.
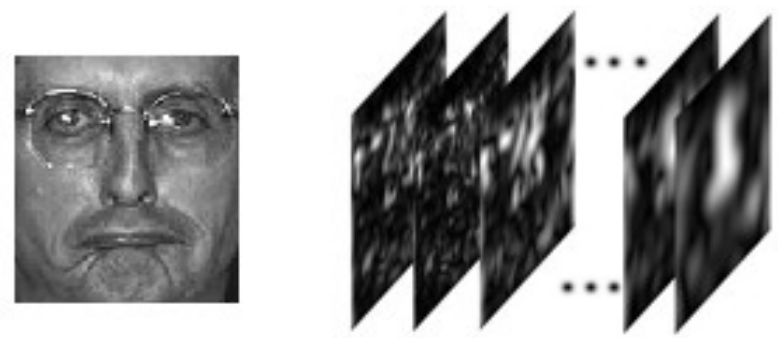

Fig. 1. A face image and its corresponding 3rd-order Gabor tensor

\section{Discriminant Analysis with Gabor Tensor Representation}

Since it is hard to apply discriminant analysis directly in the 3rd or higher-order tensor space, we here adopt an alternative strategy. The 3rd-order Gabor tensor is first unfolded into three 2nd-order tensors (matrices) along different axes. Fig. 2 2 shows the modes of the Gabor tensor unfolding. After that 2D linear/kernel discriminant analysis is conducted on these 2nd-order tensors respectively to extract effective and robust subspaces which will be combined further for classification. 


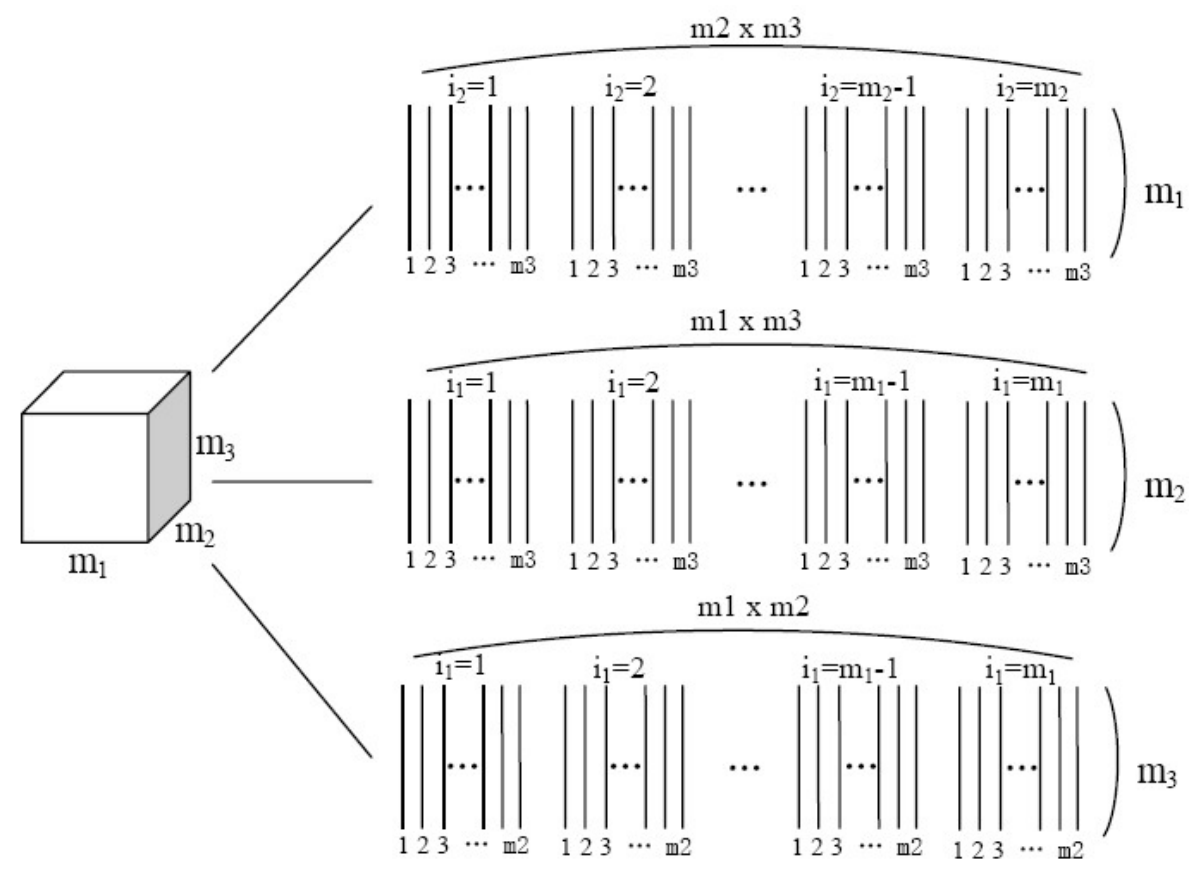

Fig. 2. Illustration of the three feature matrices unfolded from a 3rd-order tensor along different axes $\mathrm{m} 1, \mathrm{~m} 2, \mathrm{~m} 3$

\subsection{D Linear Discriminant Analysis (2D-LDA)}

2D-LDA is based on the matrices rather than vectors as opposed to 1D-LDA based approaches. Let the sample set be $X=\left\{X_{1}, X_{2}, \ldots, X_{n}\right\}$, and $X_{i}$ is an $r \times c$ unfolded Gabor matrix. The within-class scatter matrix $S_{w}$ and the between-class scatter matrix $S_{b}$ based on 2nd-order tensors are defined as follows:

$$
\begin{gathered}
S_{w}=\sum_{i=1}^{L} \sum_{X_{j} \in C_{i}}\left(X_{j}-m_{i}\right)^{T}\left(X_{j}-m_{i}\right) \\
S_{b}=\sum_{i=1}^{L} n_{i}\left(m_{i}-m\right)^{T}\left(m_{i}-m\right)
\end{gathered}
$$

where $m_{i}=\frac{1}{n_{i}} \sum_{X_{j} \in C_{i}} X_{j}$ is the mean of data matrices in class $C_{i}$, and $m=\frac{1}{n} \sum_{i=1}^{L} \sum_{X_{j} \in C_{i}}$ $X_{j}$ is the global mean matrix. The 2D-LDA searches such optimal projections that after projecting the original data onto these directions, the trace of the resulting betweenclass scatter matrix is maximized while the trace of the within-class scatter matrix is minimized. Let $W$ denote a $c \times d(d<c)$ projection matrix, and the $r \times c$ unfolded Gabor matrix $X$ is projected onto $W$ by the following linear transformation:

$$
Y=X W
$$


where the resulting $Y$ is a $r \times d$ matrix smaller than $X$. Denote $\tilde{S}_{w}, \tilde{S}_{b}$ the with-class and between-class scatter matrices of the projected data $Y$ respectively. 2D-LDA then chooses $W$ so that the following object function is maximized:

$$
J=\frac{\operatorname{tr}\left(\tilde{S}_{b}\right)}{\operatorname{tr}\left(\tilde{S}_{w}\right)}=\frac{\operatorname{tr}\left(\sum_{i=1}^{L} n_{i} W^{T}\left(m_{i}-m\right)^{T}\left(m_{i}-m\right) W\right)}{\operatorname{tr}\left(\sum_{i=1}^{L} \sum_{X_{j} \in C_{i}} W^{T}\left(X_{j}-m_{i}\right)^{T}\left(X_{j}-m_{i}\right) W\right)}=\frac{\operatorname{tr}\left(W^{T} S_{b} W\right)}{\operatorname{tr}\left(W^{T} S_{w} W\right)}
$$

The optimal projection matrix $W_{\text {opt }}$ can be obtained by solving the following generalized eigen-value problem

$$
S_{w}^{-1} S_{b} W=W \Lambda
$$

where $\Lambda$ is the diagonal matrix whose diagonal elements are eigenvalues of $S_{w}^{-1} S_{b}$.

\subsection{D Kernel Discriminant Analysis (2D-KDA)}

It is well known the face appearances may lie in a nonlinear low-dimensional manifold due to the expression and illumination variations [7]. The linear methods may not be adequate to model such a nonlinear problem. Accordingly, a 2D non-linear discriminant analysis method based on kernel trick is proposed here. Like other kernel subspace representations, such as Kernel PCA (KPCA) [11], Kernel 2DPCA [4], Kernel FDA (KFDA) [17], the key idea of 2D Kernel Discrimannt Analysis (2D-KDA) is to solve the problem of 2D-LDA in an implicit feature space $F$ which is constructed by the kernel trick:

$$
\phi: x \in R^{d} \rightarrow \phi(x) \in F
$$

Given $M$ training samples, denoted by $r \times c$ unfolded Gabor matrices $A_{k}$ $(k=1,2, \ldots, M)$. A kernel-induced mapping function maps the data vector from the original input space to a higher or even infinite dimensional feature space. The kernel mapping on matrices is defined as:

$$
\Phi(A)=\left[\phi\left(A^{1}\right)^{T}, \phi\left(A^{2}\right)^{T}, \ldots, \phi\left(A^{M}\right)^{T}\right]^{T}
$$

where $A^{i}$ is the $i$-th row vector $(1 \times c)$ of the matrix A and $\phi$ is kernel mapping function on vectors. Performing 2D-LDA in $F$ means to maximize the following Fisher discriminant function:

$$
J(W)=\operatorname{argmax} \frac{\operatorname{tr}\left(W^{T} S_{b}^{\phi} W\right)}{\operatorname{tr}\left(W^{T} S_{w}^{\phi} W\right)}
$$

where $S_{b}^{\phi}$ and $S_{w}^{\phi}$ represent the between-class scatter and the within-class scatter respectively in $F$.

$$
\begin{gathered}
S_{b}^{\phi}=\sum_{i=1}^{L} n_{i}\left(\Phi_{i}-\Phi\right)^{T}\left(\Phi_{i}-\Phi\right) \\
S_{w}^{\phi}=\sum_{i=1}^{L} \sum_{A_{j} \in C_{i}}\left(\Phi\left(A_{j}\right)-\Phi_{j}\right)^{T}\left(\Phi\left(A_{j}\right)-\Phi_{j}\right)
\end{gathered}
$$

where, $\Phi_{i}=\frac{1}{n_{i}} \sum_{j=1}^{n_{i}} \Phi\left(A_{j}\right), \Phi=\frac{1}{n} \sum_{i=1}^{L} n_{i} \Phi_{i}$. 
If we follow the conventional kernel analysis as in 1D-KFDA, there exist $r \times M$ samples to span the kernel feature space $\left\{\phi\left(A_{k}^{i}\right)^{T}, i=1, \ldots, r ; k=1, \ldots, M\right\}$, which will result in heavy computational cost for subsequent optimization procedure. To alleviate the computational cost, following [20], we use $M$ samples to approximate the kernel feature space: $\Phi_{f}=\left[\phi\left(\bar{A}_{1}\right)^{T}, \ldots, \phi\left(\bar{A}_{M}\right)^{T}\right]^{T}$, here $\bar{A}_{k}$ is the mean of the $r$ row vector of $A_{k}$. Thus, (10) can be rewritten as:

$$
J(\alpha)=\operatorname{argmax} \frac{\operatorname{tr}\left(\alpha^{T} K_{b} \alpha\right)}{\operatorname{tr}\left(\alpha^{T} K_{w} \alpha\right)}
$$

and the problem of $2 \mathrm{D}-\mathrm{KDA}$ is converted into finding the leading eigenvectors of $K_{w}^{-1} K_{b}$.

$$
\begin{gathered}
K_{b}=\sum_{i=1}^{L} n_{i}\left(z_{i}-z\right)^{T}\left(z_{i}-z\right) \\
K_{w}=\sum_{i=1}^{L} \sum_{\zeta_{j} \in C_{i}}\left(\zeta_{j}-z_{i}\right)^{T}\left(\zeta_{j}-z_{i}\right)
\end{gathered}
$$

where $\zeta_{j}=\left[\varsigma_{1 j}^{T}, \varsigma_{2 j}^{T}, \ldots, \varsigma_{r j}^{T}\right]^{T}, \varsigma_{i j}=\left[k\left(\bar{A}_{1}, A_{j}^{i}\right), k\left(\bar{A}_{2}, A_{j}^{i}\right), \ldots, k\left(\bar{A}_{M}, A_{j}^{i}\right)\right], k$ is the kernel function to compute the inner product of two vectors in $F ; z_{i}=\frac{1}{n_{i}} \sum_{\zeta_{j} \in C_{i}} \zeta_{j}$, and $z$ is the mean of all $\zeta_{j}$. Three classes of kernel functions are widely used, i.e. Gaussian kernels, polynomial kernels, and sigmoid kernels and here, we use Gaussian kernels in the following experiments.

\subsection{Discriminant Analysis with Gabor Tensor Representation}

The 2nd-order tensors, which were obtained by unfolding the 3rd-order Gabor tensor along different axes, depict faces from different aspects, so the subspaces derived from the different modes of 2nd-order tensor spaces may contain complemental information helpful for discrimination. Considering this, the subspaces are integrated and reduced further using PCA method. Suppose $Y_{1}, Y_{2}, Y_{3}$ are the three subspaces obtained for each image, they are first transformed into $1 \mathrm{D}$ vectors respectively, denoted as $y_{1}, y_{2}, y_{3}$, and concatenated into one $1 \mathrm{D}$ vector $y=\left[y_{1}^{T}, y_{2}^{T}, y_{3}^{T}\right]^{T}$, PCA is then performed on these combined vectors. Finally, the shorter vectors derived from PCA are used for classification with a nearest neighbor classifier.

As mentioned above, we proposed a novel linear/kernel discriminant analysis method with Gabor-tensor representation noted as GT-LDA and GT-KDA respectively. It inherits the advantages of the 2D discriminant analysis methods and therefore can effectively avoid the singularity problem. The algorithm is described with the Gabor tensor representation but not limit to it. In fact, it can be extended to arbitrary $N$-order tensor. Specifically, it's not hard to find that 2D-LDA and LDA are both special forms of our proposed method with $N=2$ and $N=1$.

\section{Experimental Results and Analysis}

In this section, we evaluate the performance of the proposed algorithm (GT-LDA/GTKDA) using the FERET database [10]. The FERET database is a standard test set for 


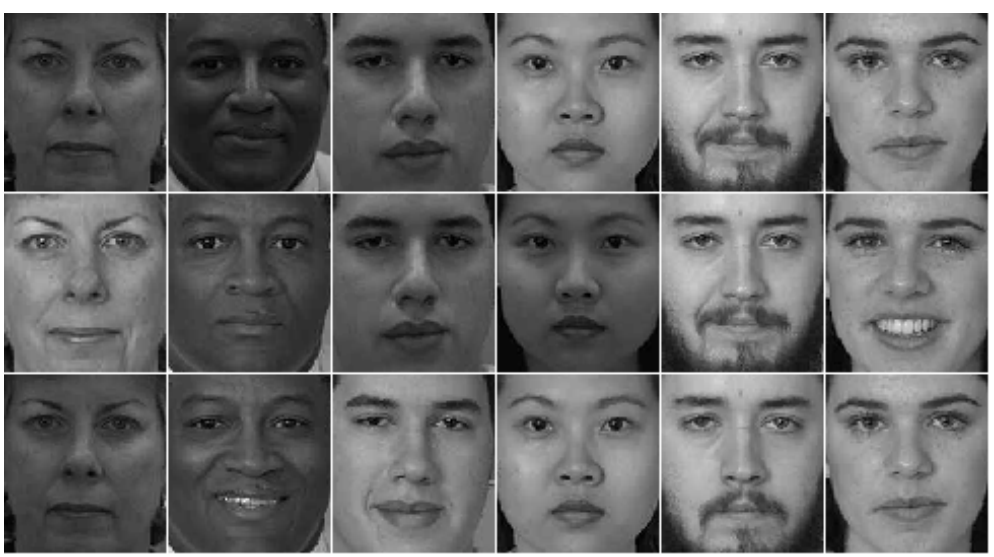

Fig. 3. Example cropped FERET images used in our experiments

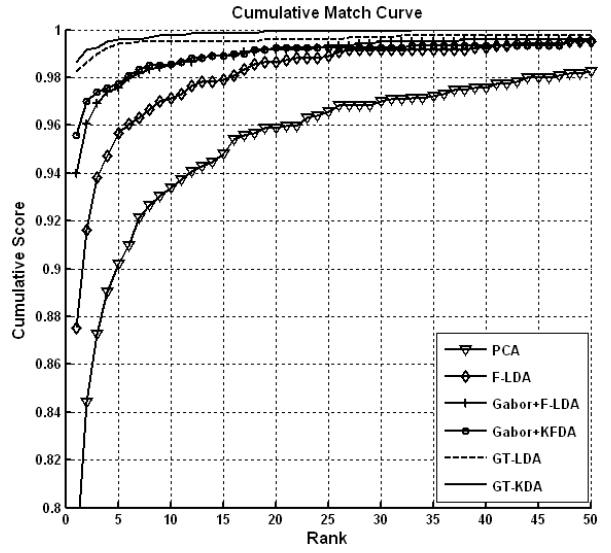

(a)

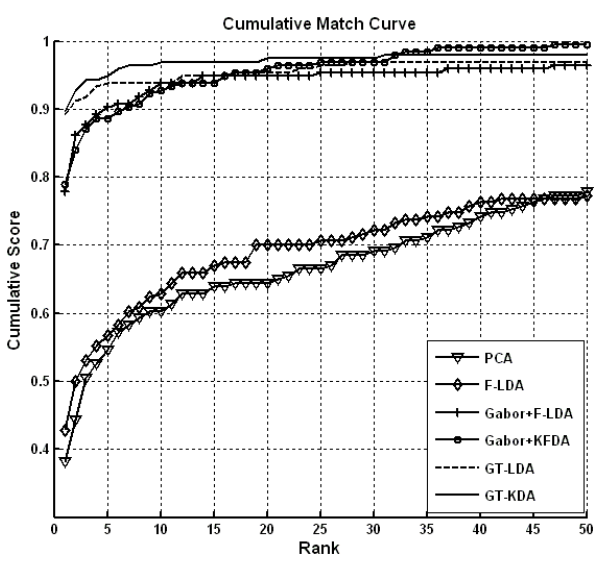

(b)

Fig. 4. Cumulative match curves on $\mathrm{fb}$ (a) and fc (b) probe sets

face recognition technologies. In our experiments, we use a subset of the training set containing 540 images from 270 subjects for training. Two probe sets named fb (expression) and fc (lighting), which contains images with expression and lighting variation respectively, are used for testing against the gallery set containing 1196 images . All images are cropped to $88 \times 80$ pixels according to the eye positions. Fig. 3 shows some examples of the cropped FERET images.

To prove the advantage of the proposed method, we compare our method (GT-LDA/ GT-KDA) with some other well-known methods: PCA, LDA, Gabor+LDA and Gabor+KFDA. Fig. 4 demonstrates the cumulative match curves (CMC) of these methods on the $\mathrm{fb}$ and $\mathrm{fc}$ probe sets and the rank-1 recognition rates are shown in Table 1 From the results, we can find the proposed method, GT-LDA and GT-KDA, all outperform 
Table 1. The rank-1 recognition rates on the FERET fb and fc probe sets

\begin{tabular}{ccc}
\hline Methods & $\mathrm{fb}($ Expression) & $\mathrm{fc}($ Lighting) \\
\hline PCA & $77.99 \%$ & $38.14 \%$ \\
F-LDA & $87.53 \%$ & $42.78 \%$ \\
Gabor+F-LDA & $93.97 \%$ & $77.84 \%$ \\
Gabor+KFDA & $95.56 \%$ & $78.87 \%$ \\
GT-LDA & $\mathbf{9 8 . 2 4 \%}$ & $\mathbf{8 9 . 1 8 \%}$ \\
GT-KDA & $\mathbf{9 8 . 6 6 \%}$ & $\mathbf{8 9 . 6 9 \%}$ \\
\hline
\end{tabular}

significantly to the other methods both in fb and fc probe sets and the kernel method, GT-KDA, achieves the best result: $98.66 \%$ in $\mathrm{fb}$ and $89.69 \%$ in fc. These results indicate the proposed method is accurate and robust to the variation of expression and illumination. Moreover, in contrast to the traditional LDA method, the computational cost of the proposed algorithm is not increased very much. In the training phase, although it needs to do three discriminant analysis rather than once in traditional LDA, the computational cost is not much higher because the $2 \mathrm{D}$ discriminant analysis is usually conducted on a lower feature space. And in the testing phase, the computational cost of the proposed method is nearly the same as the traditional LDA.

\section{Conclusions}

In this paper, a novel algorithm, linear/kenerl discriminant analysis with Gabor tensor representation has been proposed for face recognition. A face image is first encoded as a 3rd-order Gabor tensor. After unfolding the Gabor tensor and applying discriminant analysis with them, we can effectively avoid the curse of dimensionality dilemma and overcome the small sample size problem to extract different discriminative subspaces. Followed by combining these discriminant subspaces and doing reduction further, a robust and effective subspace is finally derived for face recognition. Experimental results on FERET database have shown the accuracy and robustness of the proposed method to the variation of expression and illumination.

Acknowledgements. We would like to thank Wei-shi Zheng for helpful discussion. This work was supported by the following funding resources: National Natural Science Foundation Project \#60518002, National Science and Technology Supporting Platform Project \#2006BAK08B06, National 863 Program Projects \#2006AA01Z192 and \#2006AA01Z193, Chinese Academy of Sciences 100 people project, and the AuthenMetric Collaboration Foundation.

\section{References}

1. Belhumeur, P., Hespanha, J., Kriegman, D.: Eigenfaces vs. fisherfaces: recognition using class specific linear projection. IEEE Trans. PAMI 19(7), 711-720 (1997)

2. Chen, L., Liao, H., Ko, M., Lin, J., Yu, G.: A new lda-based face recognition system which can solve the small sample size problem. Pattern Recognition (2000) 
3. Ki-Chung, C., Cheol, K.S., Ryong, K.S.: Face recognition using principal component analysis of gabor filter responses. In: Proceedings of International Workshop on Recognition, Analysis, and Tracking of Faces and Gestures in Real-Time Systems, pp. 53-57 (1999)

4. Kong, H., Li, X., Wang, L., Teoh, E.K., Wang, J.G., Venkateswarlu, R.: Generalized 2d principal component analysis. In: Proc. International Joint Conference on Neural Networks (2005)

5. Lades, M., Vorbruggen, J., Buhmann, J., Lange, J., von der Malsburg, C., Wurtz, R.P., Konen, W.: Distortion invariant object recognition in the dynamic link architecture. IEEE Transactions on Computers 42, 300-311 (1993)

6. Li, M., Yuan, B.: 2d-lda: A novel statistical linear discriminant analysis for image matrix. Pattern Recognition Letters 26(5), 527-532 (2005)

7. Li, S.Z., Jain, A.K. (eds.): Handbook of Face Recognition. Springer, New York (2005)

8. Liu, C.: Gabor-based kernel PCA with fractional power polynomial models for face recognition. IEEE Transactions on Pattern Analysis and Machine Intelligence 26(5), 572-581 (2004)

9. Liu, C., Wechsler, H.: Gabor feature based classification using the enhanced fisher linear discriminant model for face recognition. IEEE Transactions on Image Processing 11(4), 467$476(2002)$

10. Phillips, P.J., Moon, H., Rizvi, S.A., Rauss, P.J.: The FERET evaluation methodology for face-recognition algorithms. IEEE Transactions on Pattern Analysis and Machine Intelligence 22(10), 1090-1104 (2000)

11. Schölkopf, B., Smola, A., Müller, K.R.: Nonlinear component analysis as a kernel eigenvalue problem. Neural Computation 10, 1299-1319 (1999)

12. Shen, L., Bai, L.: Gabor wavelets and kernel direct disciminant analysis for face recognition. In: Int'l Conf on Pattern Recognition (ICPR'04), pp. 284-287 (2004)

13. Swets, D., Weng, J.: Using discriminant eigenfeatures for image retrieval. IEEE Trans. on PAMI 16(8), 831-836 (1996)

14. Wiskott, L., Fellous, J., Kruger, N., malsburg, C.V.: Face recognition by elastic bunch graph matching. IEEE Trans. PAMI 19(7), 775-779 (1997)

15. Xu, D., Yan, S.C., Zhang, L., Zhang, H.J., Liu, Z.K., Shum, H.Y.: Concurrent subspaces analysis. In: Proceedings of IEEE Computer Society Conference on Computer Vision and Pattern Recognition, pp. 203-208. IEEE Computer Society Press, Los Alamitos (2005)

16. Yan, S.C., Xu, D., Yang, Q., Zhang, L., Zhang, H.J.: Discriminant analysis with tensor representation. In: Proceedings of IEEE Computer Society Conference on Computer Vision and Pattern Recognition, pp. 526-532. IEEE Computer Society Press, Los Alamitos (2005)

17. Yang, M.H.: Kernel eigenface vs. kernel fisherface: Face recognition using kernel methods. In: Proc. IEEE Int. Conf on Automatic Face and Gesture Recognition. IEEE Computer Society Press, Los Alamitos (2002)

18. Ye, J., Janardan, R., Li, Q.: Two-dimensional linear discriminant analysis. In: Proceedings of Neural Information Processing Systems (2004)

19. Yu, H., Yang, J.: A direct lda algorithm for high-dimensional data with application to face recognition. Pattern Recognition (2001)

20. Zhang, D., Chen, S., Zhou, Z.: Recognizing face or object from a single image: Linear vs. kernel methods on $2 d$ patterns. In: S+SSPR06, in conjunction with ICPR06, HongKong, China (2006) 